\title{
ROBOTIC ARM CONTROLLED IN MISSILE LAUNCHERS USING PLC AND IMAGE AUTHENTICATION TECHNIQUE
}

\author{
Aaron James.S ${ }^{1}$, Sabarivani.. ${ }^{2}$, Sunitha. $\mathbf{N}^{3}$ \\ ${ }^{1}$ Assistant Professor, Department of Electronics \& Instrumentation, Sathyabama University, Tamil Nadu, India \\ ${ }^{2}$ Assistant Professor, Department of Electronics \& Instrumentation, Sathyabama University, Tamil Nadu, India \\ ${ }^{3}$ Assistant Professor, Department of Electronics \&, Instrumentation, Sathyabama University, Tamil Nadu, India
}

\begin{abstract}
As of now, the missile launchers which use Robotic Hand for holding and releasing of missiles in defense organizations are being controlled by the microcontroller. As a result of this there are many problems associated. One is that the controlling of the whole process is not perfect. Therefore we have proposed a paper where the robotic hand which controls the missile launcher will be controlled by a PLC and secured image authentication technique. In our project, the hand which is holding the missile that to be launch is controlled by the PLC. The robotic hand is operated by with the help of DC motor. The PLC controls the two kind of position of robotic hand. One is holding the missile and another one is releasing the missile. Actually the PLC is controlling the DC motor in two conditions which is holding and releasing. In the Space research center, applying the missile is very important and secure process also. So, operating with the missile launching process, secured by image authentication technique is highly required. PLC controls the robotic hand by means of ladder diagram which is nothing but programming of PLC. In that programming only our secure technique will be used. The pass code and image synchronizations are needed to execute the programming of PLC. Until the authorization the robotic hand it will not release or hold the missile.
\end{abstract}

Keywords: Robotic Hand, DC motor, PLC

\section{INTRODUCTION}

As of now, the missile launchers which use Robotic Hand for holding and releasing of missiles in defense organizations are being controlled by the microcontroller. As a result of this there are many problems associated. One is that the controlling of the whole process is not perfect. Therefore we have proposed a project where the robotic hand which controls the missile launcher will be controlled by a PLC and secured image authentication technique.

In our paper, the hand which is holding the missile that to be launch is controlled by the PLC. The robotic hand is operated by with the help of DC motor. The PLC controls the two kind of position of robotic hand. One is holding the missile and another one is releasing the missile. Actually the PLC is controlling the DC motor in two conditions which is holding and releasing. In the Space research center, applying the missile is very important and secure process also. So, operating with the missile launching process, secured by image authentication technique is highly required. PLC controls the robotic hand by means of ladder diagram which is nothing but programming of PLC. In that programming only our secure technique will be used.

\section{EXISTING SYSTEM}

Now-a-days, robotic hand model which is responsible for launching the missile in space research center and in defense sector is controlled by microcontroller. So the efficiency of controlling is not good. In present scenario the missile launchers which uses robotic arm for holding and releasing of the missile, uses microcontroller as the processing unit for the operation. As a result of this, though the making of the arrangement is cheap but there are many problems associated due to the limited scope for processing in the microcontroller. There occurs some time delay in the microcontroller and also the controlling capacity of the microcontroller for the whole process is not proper due to its limited processing scope. In the existing robotic controlled missile launchers, the hand which is holding the missile that to be launch is controlled by the microcontroller. The robotic hand is operated by with the help of DC motor. The microcontroller controls the two kind of position of robotic hand. One is holding the missile and another one is releasing the missile. This existing methodology is widely used in the defense sector and in the space research organizations. Though there may exist some other secured process in the launching of the missile like finger print authentication or palm print authentication but basically the main processing work is done by the microcontroller only. And as a result of the small scope in processing which is present in the microcontrollers, the overall controlling of the process is not perfect. There occurs some time delay and the process monitoring is not possible using any control application's like SCADA. Thus in making of the paper the existing system and the existing methodology has been thoroughly studied.

\section{PROPOSED SYSTEM}

Here in our proposed paper work the missile holding robotic hand is controlled by PLC and new techniques that are PLC pass code and Image recognition are used 
over here to make the Missile launching process fully secured. We have implemented pass code and image recognition technique in order to give the process a complete security so that there occurs no hacking or failure in launching the missiles due to the miscreants. Thus this is our proposed system.

\subsection{Proposed Methodology}

The proposed methodology or the proposed working principle is discussed below.

The process starts with entering the pass code, when the pass code is correct the web camera detects the image of the operator and if the image is correct it is sent to the MATLAB for processing, the output of the MATLAB is connected to the input of the PLC and if the image authentication is perfectly done by the MATLAB and web camera, the PLC starts processing and thus the dc motor starts which is connected to the robotic arm and thus the robotic arm does the operation of holding and releasing the missile. If the image or the pass code is wrong the buzzer sounds and the led glows. Thus it's a very highly secured process.

\subsection{Algorithm Developed}

Image authentication algorithm has been developed and is discussed below.

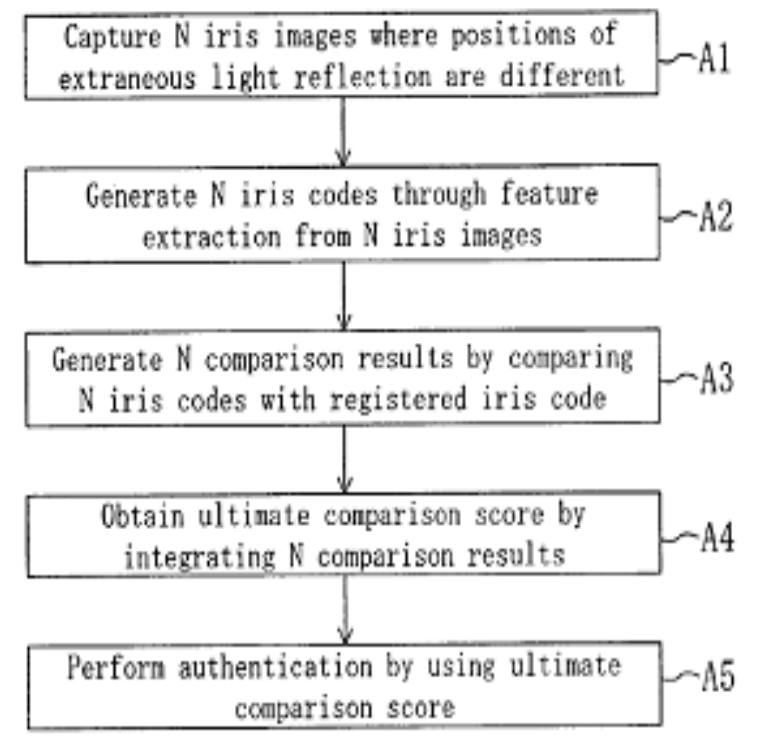

Algorithm of Image Authentication Used

The Circuit Diagram shown in the figure has The Following

1. Web camera

2. Industrial personal computer

3. Programable logic controller

4. Dc motor

5. LED

6. Buzzer

7. Robotic arm controller

8. 5 volt dc power supply
9. Relay switches

10. Single push button.

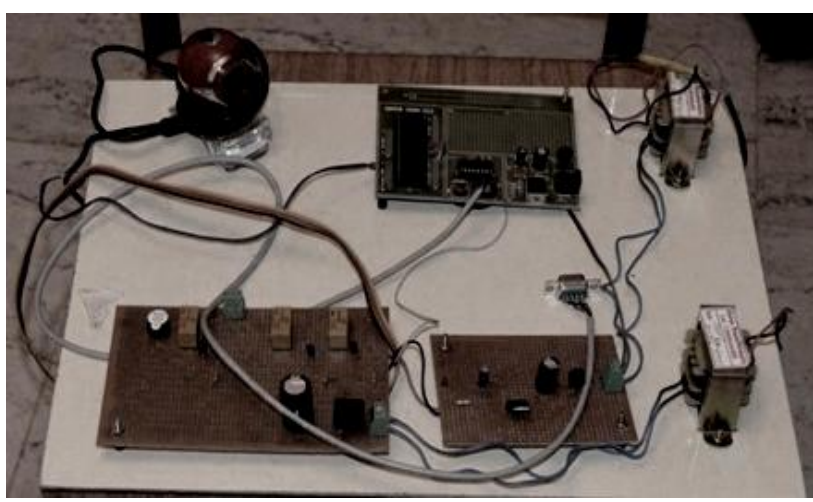

Fig.3 Circuit Diagram

\section{SOFTWARE IMPLEMENTATION}

Various Kinds of software's are being implemented in our paper work and they are discussed in great detail below. The description of the software's is discussed below.
1. PLC Language: WPL Soft V2.30
2. I/O Driver: Kepserver
3. SCADA: WonderwareIntouc
4. Image Comparisons: MATLAB

\subsection{PLC Language: WPL Soft V2.30}

Delta WPL Soft V2.30 or V.2.33 software is using for design the ladder logic for PLC. WPL Soft is a software for PLC (Programmable logic controller). When PLC is in operation, use WPL Soft to monitor the set value or temporarily saved value in timer $(\mathrm{T})$, counter $(\mathrm{C})$, and register (D) and force On/Off of output contact. WPL Soft is a program editor of Delta DVP series PLC for WINDOWS computers.

\subsection{I/O Driver Kepserver}

The EX series is the analog MPU with the lowest cost. Apart from supporting digital input and output, the EX series has built-in multiple analog I/O channels and integrates a variety of communication protocols for constructing a complete control network. EX series is suitable for all kinds of small PLC applications. MPU points: 20 (8DI/6DO, 4AI/2AO)

Max. I/O points: 238

Program capacity: 4K Steps

Communication port: Built-in RS-232 and RS-485, compatible with MODBUS ASCII / RTU communication protocol.

High-speed pulse output: Supports 2-point (Y0, Y1) independent high-speed pulse output of up to $10 \mathrm{KHz}$.

\section{Built-in 4-point high-speed counter}

Bandwidth refers to the max. counting range of a single counter. 
Built-in analog I/O function used for packaging machine, spinning machine, conveyer belt (rotation speed control), winding machine (tension control), food processing machine, landscape fountain.

\subsection{SCADA}

SCADA is an acronym for Supervisory Control and Data Acquisition. SCADA systems are used to monitor and control a plant or equipment in industries such as telecommunications, water and waste control, energy, oil and gas refining and transportation. PLCs have their origins in the automation industry and therefore are often used in manufacturing and process plant applications. The need for PLCs to connect to communication channels was not great in these applications, as they often were only required to replace traditional relay logic systems or pneumatic controllers. SCADA systems, on the other hand, have origins in early telemetry applications, where it was only necessary to know basic information from a remote source. The RTUs connected to these systems had no need for control programming because the local control algorithm was held in the relay switching logic. As PLCs were used more often to replace relay switching logic control systems, telemetry was used more and more with PLCs at the remote sites. It became desirable to influence the program within the PLC through the use of a remote signal.

\subsection{Wonderware Intouch}

SCADA is one tool for designing the process. For that, various software are used to create graphical representation of the any industrial process. Here, we are using Wonderware Intouch Software which the product coming from Invensys. Comparing other software, Intouch is cool one for designing the process. Let we see, how to create the one digital process in Wonderware Intouch SCADA software. Wonderware is the market leader in real-time operations management software.

Wonderware software delivers significant cost reductions associated with designing, building, deploying and maintaining secure and standardized applications for manufacturing and infrastructure operations.

\section{RESULTS AND DISCUSSIONS}

Missile launchers are used in defense organizations of a country and the Government gives the top most priority to its defense sector. One third of the revenue of a country is spent on its defense. Here we have designed a missile launcher which operates by involvement of programmable logic controller and image authentication technique and due to these two features its highly secured than the existing missile launchers which uses microcontroller in place of the PLC. The robotic arm will launch the missile only when the conditions of security are satisfied i.e. image authentication and pass code. Else there will be no launching of the missile by the robotic arm.

Thus initially the user enters the pass code and if it is satisfied the image authentication process starts with the use of web cam, once the image authentication is correct the PLC starts processing and the dc motor starts and the robotic arm holds or releases the missile. If the passcode or the image authentication is false than the buzzer sounds and the led glows which is a tight measure of security. We have implemented in our paper the PLC since the processing of PLC is much faster and accurate than the microcontroller in several ways. Also using one PLC we can operate many robotic missile launchers. The image authentication and passcode technique is used since they prove to be highly secured. The process starts with entering the passcode, when the passcode is correct the web camera detects the image of the operator and if the image is correct it is sent to the MATLAB for processing, the output of the MATLAB is connected to the input of the PLC and if the image authentication is perfectly done by the MATLAB and web camera, the PLC starts processing and thus the dc motor starts which is connected to the robotic arm and thus the robotic arm does the operation of holding and releasing the missile. If the image or the pass code is wrong the buzzer sounds and the led glows. Thus it's a very highly secured process.

\subsection{Performance Analysis}

In our proposed paper work the performance of the complete process is increased using PLC. On the other hand when missile launchers are handled using microcontroller the process becomes somewhat slow as compared to the PLC. Thus when the microcontroller is removed and the PLC is implemented we have analyzed that the time delay is also reduced. If the microcontroller took 2 millisecond for the operation, the PLC can do the same in 0.5 millisecond. Thus there is a reduction of 1.5 millisecond time delay in our proposed paper work. Moreover using PLC we can control the process very efficiently and thus implement SCADA and other supervisory tools for monitoring the complete process. Also we can control at least 23 missile launchers using a single standard PLC. Thus the efficiency of the complete process is increased as a whole which is not possible using microcontroller. And also with the implementation of image authentication and passcode technique in our proposed paper, the complete process became fully secured.

\subsection{Comparisons}

Comparing our proposed paper work with that of the existing robotic controlled missile launchers we have seen that the existing missile launchers which uses microcontroller for processing lacks in efficient controlling of the process and also the monitoring of the complete process is not possible. But in our proposed paper work we used PLC for controlling the robotic arm for releasing and holding of the missile and we found that the controlling is very good for the whole process as compared with the microcontroller. Moreover we can completely monitor the process using SCADA and thus process became much more secured with pass code verification and image authentication technique, which is not possible using microcontroller. Moreover using PLC also reduced the time delay as 
compared with the microcontroller which posses some time delay due to limited processing scope.

\section{Robotic Arm with Missile}

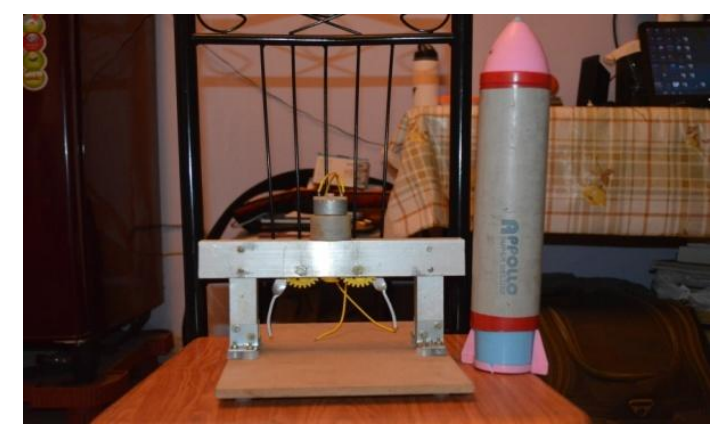

\section{Missile Being Released}

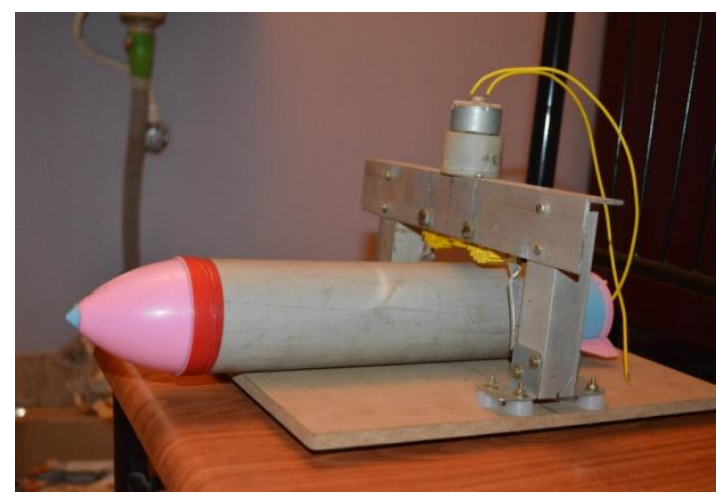

\section{CONCLUSIONS AND FUTURE WORK}

The Robotic arm controlled in missile launchers using PLC and image authentication technique is carried out in prototype model as discussed. Thus the design is successful and the predicted output is also obtained in a perfect way without any much complications. Thus the robotic arm is functioning on the command of the PLC and the MATLAB and the process is being supervised using SCADA thereby avoiding monitoring and controlling complications.In the present situation of the missile launchers the microcontroller is used. To reduce the drawbacks of the microcontroller and human errors we need to have some kind of high processing device and the PLC is most fit for it. Also for high security and for hacking proof reasons we implemented the image authentication and the pass code technique in our paper so that it proves to be a highly secured missile launcher using PLC and image authentication technique.

\section{REFERENCES}

[1] Ching-Yung Lin, Member, IEEE, and Shih-Fu Chang, Member IEEE "A Robust Image Authentication Method Distinguishing JPEG Compression from Malicious Manipulation", Vol. No : 23 ${ }^{\text {rd }}, 2010$, ISBN 13383, pp. 43-85.

[2] S. K. Nayak, Dr. S. B. Thorat, Miss. Jyoti P Dandale "Facial Recognition Technology : An analysis with scope in India", Vol. No. : 28, 2006, ISBN 19987, pp. 28-43.
[3] A semiautonomous sprawl robot based on remote wireless control,HongKai Li; ZhenDong Dai;Inst. of Bio-inspired Struct. \&Surface Eng., Nanjing Univ. of Aeronaut. \& Astronaut., Nanjing,China,

[4] Camera-in-hand robotic system for remote monitoring of plantgrowth in a laboratory , Seelye, M.; Sen Gupta, G.; Seelye,J.; Mukhopadhyay, S.C.; Sch. of Eng. \& Adv. Technol. (SEAT),Massey Univ., Palmerston North, New Zealand, This paper appears in: Instrumentation and Measurement Technology Conference (I2MTC), 2010 IEEE ,Issue Date: 3-6 May 2010

[5] Multifunctional Sensor Networks for Automation, ProcessMonitoring and Robotic Applications , BalakrishnanG.; Hiremath, S.S.; Dept. of Mech. Eng., Indian Inst. of Technol.Madras, Chennai, India,This paper appears in: Robotics inAlpe-Adria-Danube Region (RAAD), 2010 IEEE 19thInternational Workshop on Issue Date: 24-26 June 2010 